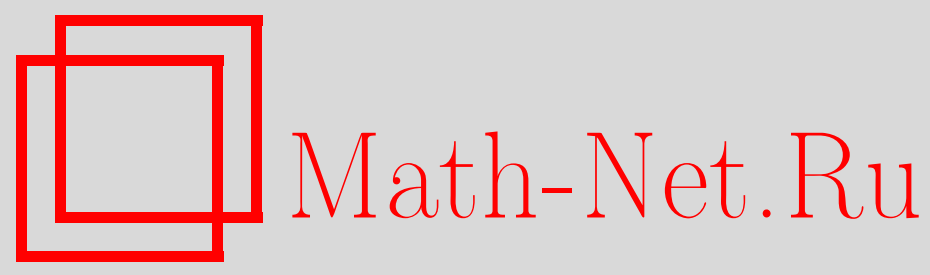

В. А. Краснов, Вещественные алгебраически максимальные многообразия, Матем. заметки, 2003, том 73, выпуск $6,853-860$

DOI: https://doi.org/10.4213/mzm233

Использование Общероссийского математического портала Math-Net.Ru подразумевает, что вы прочитали и согласны с пользовательским соглашением http://www . mathnet.ru/rus/agreement

Параметры загрузки:

IP: 54.162 .127 .20

26 апреля 2023 г., $15: 34: 47$

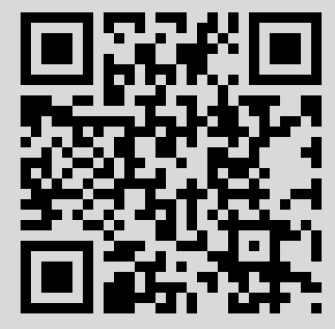




\section{ВЕЩЕСТВЕННЫЕ АЛГЕБРАИЧЕСКИ МАКСИМАЛЬНЫЕ МНОГООБРАЗИЯ}

\section{В. А. Краснов}

Для вещественных алгебраических многообразий, у которых вещественная алгебраическая группа когомологий максимальна, строится канонический гомоморфизм из группы когомологий множества комплексных точек в группу когомологий множества вещественных точек, а затем доказывается, что этот гомоморфизм является изоморфизмом.

Библиограффия: 5 названий.

Введение. Далее $X$ - неособое $n$-мерноепроективное вещественное алгебраическое многообразие, т.е. $X$ - такая схема над $\mathbb{R}$, что произведение $X \otimes \mathbb{C}$ является $n$-мерным неособым проективным комплексным алгебраическим многообразием. Множество комплексных точек $X(\mathbb{C})$ является компактным комплексньп $n$-мерньм многообразием, а множество вещественных точек $X(\mathbb{R})$, если оно непустое, является $n$-мерным компактным дифференцируемым многообразием. Пусть $A^{*}(X)$ - кольцо Чжоу многообразия $X$, тогда определено комплексное отображение цикла

$$
\operatorname{cl}_{\mathbb{C}}: A^{*}(X) \rightarrow H^{*}\left(X(\mathbb{C}), \mathbb{F}_{2}\right),
$$

которое циклу $Z \in A^{q}(X)$ сопоставляет класс когомологий

$$
[Z(\mathbb{C})]^{*} \in H^{2 q}\left(X(\mathbb{C}), \mathbb{F}_{2}\right) .
$$

Образ этого отображения обозначим через $H_{\mathrm{ra}}^{*}\left(X(\mathbb{C}), \mathbb{F}_{2}\right)$ и назовем вещественной алгебраической группой когомологий. Если выполняется равенство

$$
H_{\mathrm{ra}}^{*}\left(X(\mathbb{C}), \mathbb{F}_{2}\right)=H^{*}\left(X(\mathbb{C}), \mathbb{F}_{2}\right),
$$

то многообразие $X$ будем назьвать алгебраически максимальным многообразием $(A M$-многообразием). Кроме комплексного отображения цикла $(0.1)$ определено вещественное отображение цикла

$$
\operatorname{cl}_{\mathbb{R}}: A^{*}(X) \rightarrow H^{*}\left(X(\mathbb{R}), \mathbb{F}_{2}\right)
$$

которое циклу $Z \in A^{q}(X)$ сопоставляет класс когомологий

$$
[Z(\mathbb{R})]^{*} \in H^{q}\left(X(\mathbb{R}), \mathbb{F}_{2}\right) .
$$

В этой заметке доказьвается следующая

Работа частично поддержана фондом INTAS, грант INTAS-OPEN-2000-268. 
Теорема 0.1. Пусть $X$ является алгебраически максимальным многообразием, тогда справедливы следующие утвержсдения:

1) для каждого $0 \leqslant q \leqslant \operatorname{dim} X$ однозначно определен гомоморфизм когомоло2uั̆

$$
\gamma^{q}: H^{2 q}\left(X(\mathbb{C}), \mathbb{F}_{2}\right) \rightarrow H^{q}\left(X(\mathbb{R}), \mathbb{F}_{2}\right)
$$

который удовлетворяет условию $\gamma^{q} \circ \mathrm{cl}_{\mathbb{C}}=\mathrm{cl}_{\mathbb{R}}$

2) при каждом $q$ гомоморфизм $\gamma^{q}$ является изоморфизмом.

1. Эквивариантные когомологии. При доказательстве теоремы 0.1 мы будем применять эквивариантные когомологии и эквивариантное отображение цикла. В этом пункте приводятся без доказательства необходимые далее факты об эквивариантных когомологиях вешественного алгебраического многообразия. Подробности можно посмотреть в [1]-[3].

На множестве комплексных точек $X(\mathbb{C})$ действует группа Галуа $G=G(\mathbb{C} / \mathbb{R})$, поэтому определены эквивариантные когомологии $H^{*}\left(X(\mathbb{C}) ; G, \mathbb{F}_{2}\right)$. Имеются две спектральные последовательности, которые сходятся к этим когомологиям. Первая спектральная последовательность нам не потребуется, поэтому мы ее не приводим, а вторая спектральная последовательность имеет вид

$$
I I_{2}^{p, q}=H^{p}\left(G, H^{q}\left(X(\mathbb{C}), \mathbb{F}_{2}\right)\right) \Longrightarrow H^{p+q}\left(X(\mathbb{C}) ; G, \mathbb{F}_{2}\right)
$$

Имеется несколько критериев того, что эта спектральная последовательность вырождается (см. [3]). Два из них нам потребуются.

КРИТЕРИй 1. Спектральная последовательность (1.1) вырождается тогда и только тогда, когда вьполняется равенство

$$
\operatorname{dim} H^{*}\left(X(\mathbb{R}), \mathbb{F}_{2}\right)=\operatorname{dim} H^{1}\left(G, H^{*}\left(X(\mathbb{C}), \mathbb{F}_{2}\right)\right)
$$

КРИТЕРИй 2. Спектральная последовательность (1.1) вырождается тогда и только тогда, когда образ канонического гомоморфизма (забывания вещественной структуры)

$$
\alpha: H^{q}\left(X(\mathbb{C}) ; G, \mathbb{F}_{2}\right) \rightarrow H^{q}\left(X(\mathbb{C}), \mathbb{F}_{2}\right)
$$

равен инвариантной групе $H^{q}\left(X(\mathbb{C}), \mathbb{F}_{2}\right)^{G}$ при $0 \leqslant q \leqslant 2 \operatorname{dim} X$.

Заметим, что равенство (1.2) по определению означает, что $X$ является $G M$-многообразием (Галуа максимальным многообразием).

Обозначим через $t$ образующую группы

$$
H^{1}\left(\mathrm{pt} ; G, \mathbb{F}_{2}\right)=H^{1}\left(G, \mathbb{F}_{2}\right)
$$

где $\mathrm{pt}$ - одноточечное $G$-пространство. Тогда гомоморфизм умножения на $t$ будем обозначать через

$$
t: H^{q}\left(X(\mathbb{C}) ; G, \mathbb{F}_{2}\right) \rightarrow H^{q+1}\left(X(\mathbb{C}) ; G, \mathbb{F}_{2}\right)
$$

и называть гомоморфизмом сдвига (см. [3]). 
Кроме комплексного (0.1) и вещественного (0.2) отображений цикла определено эквивариантное отображение цикла

$$
\mathrm{cl}: A^{*}(X) \rightarrow H^{*}\left(X(\mathbb{C}) ; G, \mathbb{F}_{2}\right),
$$

которое циклу $Z \in A^{q}(X)$ сопоставляет эквивариантньй класс когомологий

$$
[Z(\mathbb{C}) ; G]^{*} \in H^{2 q}\left(X(\mathbb{C}) ; G, \mathbb{F}_{2}\right) \text {. }
$$

Эти отображения цикла связаны между собой соотношениями

$$
\alpha \circ \mathrm{cl}=\operatorname{cl}_{\mathbb{C}}, \quad \beta \circ \mathrm{cl}=\mathrm{Sq}_{\mathrm{Cl}} \circ \mathrm{cl}_{\mathbb{R}}
$$

где

$$
\beta: H^{2 q}\left(X(\mathbb{C}) ; G, \mathbb{F}_{2}\right) \rightarrow H^{2 q}\left(X(\mathbb{R}) ; G, \mathbb{F}_{2}\right)=\bigoplus_{k=0}^{2 q} H^{k}\left(X(\mathbb{R}), \mathbb{F}_{2}\right)
$$

- гомоморфизм ограничения, a $\mathrm{Sq}$ - полньй квадрат Стинрода.

Напомним, что многообразие $X$ назьвается $M$-многообразием, если выполняется равенство

$$
\left.\operatorname{dim} H^{*}\left(X(\mathbb{R}), \mathbb{F}_{2}\right)=\operatorname{dim} H^{*}\left(X(\mathbb{C}), \mathbb{F}_{2}\right)\right) .
$$

Для того чтобы $X$ было $M$-многообразием, необходимо и достаточно, чтобы $X$ было $G M$-многообразием и инволюция комплексного сопряжения $g \in G$ действовала на группе когомологий $H^{*}\left(X(\mathbb{C}), \mathbb{F}_{2}\right)$ тривиально. Для $M$-многообразия гомоморфизм ограничения

$$
\beta: H^{*}\left(X(\mathbb{C}) ; G, \mathbb{F}_{2}\right) \rightarrow H^{*}\left(X(\mathbb{R}) ; G, \mathbb{F}_{2}\right)
$$

мономорфен.

2. Доказательство основной теоремы. Сначала докажем несколько лемм.

ЛЕмма 2.1. Кажсдое алгебраически максимальное многообразие является $M$ многообразием.

ДокАЗАТЕЛЬСТво. Для алгебраически максимального многообразия $X$ вьполняются равенства

$$
\alpha\left(\operatorname{cl}\left(A^{q}(X)\right)\right)=\operatorname{cl}_{\mathbb{C}}\left(A^{q}(X)\right)=H_{\mathrm{ra}}^{2 q}\left(X(\mathbb{C}), \mathbb{F}_{2}\right)=H^{2 q}\left(X(\mathbb{C}), \mathbb{F}_{2}\right)
$$

Следовательно, выполняется условие $G M$-максимальности из второго критерия и инволюция комплексного сопряжения $g \in G$ действует на группе когомологий $H^{*}\left(X(\mathbb{C}), \mathbb{F}_{2}\right)$ тривиально. Лемма доказана.

ЛЕмма 2.2. Для алгебраически максимального многообразия $X$ выполняются при $0 \leqslant q \leqslant \operatorname{dim} X$ равенства

$$
H^{2 q}\left(X(\mathbb{R}) ; G, \mathbb{F}_{2}\right)=\operatorname{cl}\left(A^{q}(X)\right)+t^{2}\left(\operatorname{cl}\left(A^{q-1}(X)\right)\right)+\cdots+t^{2 q}\left(\operatorname{cl}\left(A^{0}(X)\right)\right) .
$$


ДокАЗАТЕЛЬСТво. Мы будем проверять это равенство индукцией по $q$. При $q=0$ оно очевидно. Пусть это равенство вьполняется при $q=k$; покажем, что тогда оно выполняется при $q=k+1$. Применим к равенству

$$
H^{2 k}\left(X(\mathbb{R}) ; G, \mathbb{F}_{2}\right)=\operatorname{cl}\left(A^{k}(X)\right)+t^{2}\left(\operatorname{cl}\left(A^{k-1}(X)\right)\right)+\cdots+t^{2 k}\left(\operatorname{cl}\left(A^{0}(X)\right)\right)
$$

гомоморфизм сдвига $t^{2}$, тогда получим равенство

$$
t^{2}\left(H^{2 k}\left(X(\mathbb{R}) ; G, \mathbb{F}_{2}\right)\right)=t^{2}\left(\operatorname{cl}\left(A^{k}(X)\right)\right)+\cdots+t^{2 k+2}\left(\operatorname{cl}\left(A^{0}(X)\right)\right) .
$$

Так как

$$
\alpha\left(\operatorname{cl}\left(A^{k+1}(X)\right)\right)=H^{2 k+2}\left(X(\mathbb{C}), \mathbb{F}_{2}\right),
$$

то из второй спектральной последовательности следует, что выполняется равенство

$$
H^{2 k+2}\left(X(\mathbb{C}) ; G, \mathbb{F}_{2}\right)=\operatorname{cl}\left(A^{k+1}(X)\right)+t^{2}\left(H^{2 k}\left(X(\mathbb{R}) ; G, \mathbb{F}_{2}\right)\right) .
$$

Следовательно,

$$
\left.H^{2 k+2}\left(X(\mathbb{C}) ; G, \mathbb{F}_{2}\right)\right)=\operatorname{cl}\left(A^{k+1}\right)+t^{2}\left(\operatorname{cl}\left(A^{k}(X)\right)\right)+\cdots+t^{2 k+2}\left(\operatorname{cl}\left(A^{0}(X)\right)\right) .
$$

Лемма доказана.

ЛЕмма 2.3. Для алгебраически максимального многообразия $X$ и чикла $Z \in$ $A^{q}(X)$ справедливо утверждение: если $\operatorname{cl}_{\mathbb{C}}(Z)=0$, то также $\operatorname{cl}_{\mathbb{R}}(Z)=0$.

ДокАЗАТЕЛЬСТво. $\operatorname{Eсли~} \mathrm{cl}_{\mathbb{C}}(Z)=0$, то

$$
z=\operatorname{cl}(Z) \in t^{2}\left(H^{2 q-2}\left(X(\mathbb{C}) ; G, \mathbb{F}_{2}\right)\right) .
$$

Поэтому из леммы 2.2 следует, что существуют циклы

$$
Z_{0} \in A^{0}(X), \quad Z_{1} \in A^{1}(X), \quad \ldots, \quad Z_{q-1} \in A^{q-1}(X)
$$

такие, что вьполняется равенство

$$
z=t^{2 q}\left(\operatorname{cl}\left(Z_{0}\right)\right)+t^{2 q-2}\left(\operatorname{cl}\left(Z_{1}\right)\right)+\cdots+t^{2}\left(\operatorname{cl}\left(Z_{q-1}\right)\right) .
$$

Следовательно,

$$
t^{2 q}\left(\operatorname{cl}\left(Z_{0}\right)\right)+t^{2 q-2}\left(\operatorname{cl}\left(Z_{1}\right)\right)+\cdots+t^{2}\left(\operatorname{cl}\left(Z_{q-1}\right)\right)+\operatorname{cl}(Z)=0 .
$$

Поэтому,

$$
\begin{gathered}
\beta\left(t^{2 q}\left(\operatorname{cl}\left(Z_{0}\right)\right)+t^{2 q-2}\left(\operatorname{cl}\left(Z_{1}\right)\right)+\cdots+t^{2}\left(\operatorname{cl}\left(Z_{q-1}\right)\right)+\operatorname{cl}(Z)\right) \\
\left.=\operatorname{Sq}_{\left(c_{\mathbb{R}}\right.}\left(Z_{0}\right)+\operatorname{cl}_{\mathbb{R}}\left(Z_{1}\right)+\cdots+\operatorname{cl}_{\mathbb{R}}\left(Z_{q-1}\right)+\operatorname{cl}_{\mathbb{R}}(Z)\right)=0, \\
\operatorname{cl}_{\mathbb{R}}\left(Z_{0}\right)+\operatorname{cl}_{\mathbb{R}}\left(Z_{1}\right)+\cdots+\operatorname{cl}_{\mathbb{R}}\left(Z_{q-1}\right)+\operatorname{cl}_{\mathbb{R}}(Z)=0 .
\end{gathered}
$$

Осталось заметить, что

$$
\begin{array}{r}
\operatorname{cl}_{\mathbb{R}}\left(Z_{0}\right) \in H^{0}\left(X(\mathbb{R}), \mathbb{F}_{2}\right), \quad \operatorname{cl}_{\mathbb{R}}\left(Z_{1}\right) \in H^{1}\left(X(\mathbb{R}), \mathbb{F}_{2}\right), \quad \ldots, \\
\operatorname{cl}_{\mathbb{R}}\left(Z_{q-1}\right) \in H^{q-1}\left(X(\mathbb{R}), \mathbb{F}_{2}\right), \quad \operatorname{cl}_{\mathbb{R}}(Z) \in H^{q}\left(X\left(\mathbb{R}, \mathbb{F}_{2}\right) .\right.
\end{array}
$$

Лемма доказана.

Перейдем непосредственно к доказательству теоремы. 
ДоКАТЕЛЬСТво теОРемЫ 0.1. Гомоморфизм

$$
\gamma^{q}: H^{2 q}\left(X(\mathbb{C}), \mathbb{F}_{2}\right) \rightarrow H^{q}\left(X(\mathbb{R}), \mathbb{F}_{2}\right)
$$

определяется следующим образом. Пусть

$$
z \in H^{2 q}\left(X(\mathbb{C}), \mathbb{F}_{2}\right), \quad Z \in A^{q}(X)
$$

- такие элементы, что $z=\operatorname{cl}_{\mathbb{C}}(Z)$. Тогда положим $\gamma^{q}(z)=\operatorname{cl}_{\mathbb{R}}(Z)$. Из леммы 2.3 следует, что определение элемента $\gamma^{q}(z)$ зависит только от класса когомологий $z \in H^{2 q}(X(\mathbb{C})$ ). Поэтому гомоморфизм $\gamma^{q}$ определен корректно и удовлетворяет условию

$$
\gamma^{q} \circ \mathrm{cl}_{\mathbb{C}}=\mathrm{cl}_{\mathbb{R}} .
$$

Покажем теперь, что гомоморфизм $\gamma^{q}$ является мономорфизмом. Действительно, если $\gamma^{q}(z)=\mathrm{cl}_{\mathbb{R}}(Z)=0$, то $\beta(\operatorname{cl}(Z))=0$. Но для $M$-многообразия гомоморфизм $\beta$ мономорфен, следовательно, $z=\operatorname{cl}_{\mathbb{C}}(Z)=\alpha(\operatorname{cl}(Z))=0$.

Остается заметить, что из равенства

$$
\left.\operatorname{dim} H^{*}\left(X(\mathbb{R}), \mathbb{F}_{2}\right)=\operatorname{dim} H^{*}\left(X(\mathbb{C}), \mathbb{F}_{2}\right)\right)
$$

следует эпиморфность гомоморфизма $\gamma^{q}$. Теорема доказана.

3. Замечания и примеры. Примером алгебраически максимального многообразия служит многообразие, обладающее клеточным разложением. Напомним, что неособое вещественное проективное алгебраическое многообразие $X$ обладает клеточныцм разложением, если существует фильтрация

$$
X=X_{m} \supset X_{m-1} \supset \cdots \supset X_{0} \supset X_{-1}=\varnothing
$$

замкнутыми подсхемами, причем каждая разность $X_{i} \backslash X_{i-1}$ представляет собой объединение схем $U_{i j}$, изоморфных аффинным пространствам $\mathbb{A}^{n_{i j}}$. Например, многообразие Грассмана обладает клеточным разложением. Для многообразия $X$ с клеточным разложением имеет место следуюшее

УТВеРЖДЕНИЕ. Если $V_{i j}$ - замыкание $U_{i j}$, то классы рациональной эквивалентности $\left[V_{i j}\right]$ дают аддитивный базис кольиа Чжоу $A^{*}(X)$, причем отображение иикла

$$
\operatorname{cl}_{\mathbb{C}}^{\mathbb{Z}}: A^{*}(X) \rightarrow H^{*}(X(\mathbb{C}), \mathbb{Z})
$$

является изоморфизмом.

Следовательно, гомоморфизм

$$
\operatorname{cl}_{\mathbb{C}}: A^{*}(X) \rightarrow H^{*}\left(X(\mathbb{C}), \mathbb{F}_{2}\right)
$$

эпиморфен, а поэтому $X$ является алгебраически максимальным. Впрочем, утверждение нашей теоремы для многообразия с клеточньм разложением очевидно, поэтому желательно иметь примеры алгебраически максимальных многообразий, не обладающих клеточным разложением. 
Если $X$ - алгебраически максимальноемногообразие, а $Y \subset X$ - алгебраически максимальное подмногообразие, то при моноидальном преобразовании $X$ с центром $Y$ получится алгебраически максимальное многообразие. Пусть, например, $X$ получается из вещественной проективной плоскости $P^{2}$ с помощью $\sigma$-процесса с центрами в трех вещественньх точках, не лежащих на одной прямой. Тогда $X$ является алгебраически максимальной поверхностью, не обладающей клеточным разложением.

Сделаем несколько замечаний про топологические свойства множества вещественных точек $X(\mathbb{R})$ алгебраически максимального многообразия. Если $n=\operatorname{dim} X$, то

$$
\operatorname{dim} H^{n}\left(X(\mathbb{R}), \mathbb{F}_{2}\right)=\operatorname{dim} H^{2 n}\left(X(\mathbb{C}), \mathbb{F}_{2}\right)=\mathbb{F}_{2} .
$$

Поэтому многообразие $X(\mathbb{R})$ состоит из одной компоненты. Пусть $K_{X} \in A^{1}(X)$ - канонический класс многообразия, тогда

$$
\begin{aligned}
& \operatorname{cl}_{\mathbb{C}}\left(K_{X}\right)=c_{1}(X(\mathbb{C}))(\bmod 2) \in H^{2}\left(X(\mathbb{C}), \mathbb{F}_{2}\right), \\
& \operatorname{cl}_{\mathbb{R}}\left(K_{X}\right)=w_{1}(X(\mathbb{R})) \in H^{1}\left(X(\mathbb{R}), \mathbb{F}_{2}\right) .
\end{aligned}
$$

Поэтому многообразие $X(\mathbb{R})$ ориентируемое тогда и только тогда, когда

$$
c_{1}(X(\mathbb{C}))(\bmod 2)=0 .
$$

Отметим еще, что для эйлеровой характеристики вьполняется равенство $\chi(X(\mathbb{R}))=$ $\sigma(X(\mathbb{C}))$, где $\sigma(X(\mathbb{C}))$ - сигнатура многообразия $X(\mathbb{C})$. Это равенство выводится из формулы Лефшеца для инволюции комплексного сопряжения.

4. Одно приложение. Через $Z_{\sigma}^{q}(X), q \in(-\infty, n)$, обозначим группу сингулярных циклов на $X$. Ееэлементами являются линейные комбинации с целыми коэффициентами $m_{1} F_{1}+\cdots+m_{k} F_{k}$, где $F_{i}: M_{i} \rightarrow X, i=1, \ldots, k$, -отображения неособых $(n-q)$-мерных вещественньх алгебраических многообразий в $X$. Через

$$
M U^{2 q}(X(\mathbb{C})), \quad M O^{q}(X(\mathbb{R}))
$$

обозначаются группа комплексных кобордизмов многообразия $X(\mathbb{C})$ и групша неориентированных кобордизмов многообразия $X(\mathbb{R})$ соответственно. Определены отображения цикла

$$
\begin{aligned}
& \mathrm{CL}_{\mathbb{C}}: Z_{\sigma}^{q}(X) \rightarrow M U^{2 q}(X(\mathbb{C})), \\
& \mathrm{CL}_{\mathbb{R}}: Z_{\sigma}^{q}(X) \rightarrow M O^{q}(X(\mathbb{R})) .
\end{aligned}
$$

В работе [4] было доказано

ПРЕДЛОЖЕНИЕ 4.1. Пусть многообразие $X$ обладает клеточным разложением и является $M$-многообразием. Тогда для кажсого $q \in \mathbb{Z}$ существует гомомор$\oint и з м$

$$
\sigma_{q}: M U^{2 q}(X(\mathbb{C})) \rightarrow M O^{q}(X(\mathbb{R})),
$$

который определяется равенством

$$
\sigma_{q}\left(\mathrm{CL}_{\mathbb{C}}(F)\right)=\mathrm{CL}_{\mathbb{R}}(F)
$$

əде $F: M^{q+n} \rightarrow X$ - произвольное отображсение вещественных алгебраических многообразий. 
В данной заметке мы докажем аналог предложения 4.1 для более широкого класса многообразий. А именно, рассмотрим отображение цикла кольца Чжоу в целочисленные когомологии

$$
\operatorname{cl}_{\mathbb{C}}^{\mathbb{Z}}: A^{*}(X) \rightarrow H^{*}(X(\mathbb{C}), \mathbb{Z}) .
$$

Если оно эпиморфно, то многообразие $X$ назовем $\mathbb{Z}$-алгебраически максимальным многообразием (Z्Z - $A M$-многообразием). Это понятие аналогично понятию алгебраически максимального многообразия, введенному ранее. Заметим, что многообразие, обладающее клеточным разложением, является $\mathbb{Z}$-алгебраически максимальным многообразием, а каждое $\mathbb{Z}$-алгебраически максимальное многообразие является просто алгебраически максимальным многообразием, которое в свою очередь является $M$-многообразием.

В качестве приложения мы докажем сейчас

ПРЕДЛОЖЕНИЕ 4.2. Пусть многообразие $X$ является $\mathbb{Z}-А М$-многообразием. Тогда для каждого $q \in \mathbb{Z}$ существует эпиморфизм

$$
\sigma_{q}: M U^{2 q}(X(\mathbb{C})) \rightarrow M O^{q}(X(\mathbb{R}))
$$

который удовлетворяет следующему условию: если $F: M^{q+n} \rightarrow X-$ отображение вещественных алгебраических многообразий, то

$$
\sigma_{q}\left(\mathrm{CL}_{\mathbb{C}}(F)\right)=\mathrm{CL}_{\mathbb{R}}(F)
$$

ДоКАЗАТЕЛЬСТво. Группа сингулярных циклов $Z_{\sigma}^{*}(X)$ является свободным модулем над кольцом $Z_{\sigma}^{*}(\operatorname{Spec} \mathbb{R})$. Кольцо кобордизмов точки $M U^{*}(\mathrm{pt})$ является кольцом многочленов

$$
\mathbb{Z}\left[t_{0}, t_{1}, t_{2}, \ldots\right]
$$

где $t_{i} \in M U^{-2 i}(\mathrm{pt})$, причем гомоморфизм цикла

$$
\mathrm{CL}_{\mathbb{C}}: Z_{\sigma}^{*}(\operatorname{Spec} \mathbb{R}) \rightarrow M U^{*}(\mathrm{pt})
$$

эпиморфен (см. [5]). Пусть $C_{1}, \ldots, C_{N} \in Z_{\sigma}^{*}(X)$ - такой набор сингулярных циклов, что классы когомологий

$$
\operatorname{cl}_{\mathbb{C}}^{\mathbb{Z}}\left(C_{1}\right), \ldots, \operatorname{cl}_{\mathbb{C}}^{\mathbb{Z}}\left(C_{N}\right) \in H^{*}(X(\mathbb{C}), \mathbb{Z})
$$

образуют аддитивньй базис этой свободной грушы когомологий. Тогда классы кобордизмов

$$
\mathrm{CL}_{\mathbb{C}}\left(C_{1}\right), \ldots, \mathrm{CL}_{\mathbb{C}}\left(C_{N}\right) \in M U^{*}(X(\mathbb{C}))
$$

являются свободньми образующими модуля $M U^{*}(X(\mathbb{C}))$ над кольцом кобордизмов точки $M U^{*}(\mathrm{pt})($ см. [5]).

Кольцо кобордизмов точки $M O^{*}(\mathrm{pt})$ является кольцом многочленов

$$
\mathbb{F}_{2}\left[x_{0}, x_{2}, x_{4}, x_{5}, x_{6} \ldots\right]
$$

где $x_{i} \in M O^{-i}(\mathrm{pt})$, причем $i$ не имеет вид $2^{s}-1$, а гомоморфизм цикла

$$
\mathrm{CL}_{\mathbb{R}}: Z_{\sigma}^{*}(\operatorname{Spec} \mathbb{R}) \rightarrow M O^{*}(\mathrm{pt})
$$


эпиморфен (см. [5]). Определен канонический эпиморфизм колец

$$
\sigma_{*}: M U^{*}(\mathrm{pt}) \rightarrow M O^{*}(\mathrm{pt})
$$

удовлетворяющий равенству $\sigma_{*} \circ \mathrm{CL}_{\mathbb{C}}=\mathrm{CL}_{\mathbb{R}}$ (см. [4]). Из теоремы 0.1 следует, что классы когомологий

$$
\operatorname{cl}_{\mathbb{R}}\left(C_{1}\right), \ldots, \operatorname{cl}_{\mathbb{R}}\left(C_{N}\right) \in H^{*}\left(X(\mathbb{R}), \mathbb{F}_{2}\right)
$$

образуют базис пространства $H^{*}\left(X(\mathbb{R}), \mathbb{F}_{2}\right)$ над полем $\mathbb{F}_{2}$. Следовательно, классы кобордизмов

$$
\mathrm{CL}_{\mathbb{R}}\left(C_{1}\right), \ldots, \mathrm{CL}_{\mathbb{R}}\left(C_{N}\right) \in M O^{*}(X(\mathbb{R}))
$$

являются свободньми образующими модуля $M O^{*}(X(\mathbb{R}))$ над кольцом кобордизмов точки $M O^{*}(\mathrm{pt})($ см. [5]).

Остается положить

$$
\sigma_{*}\left(\sum_{1}^{N} f_{i} \cdot \mathrm{CL}_{\mathbb{C}}\left(C_{i}\right)\right)=\sum_{1}^{N} \sigma_{*}\left(f_{i}\right) \cdot \mathrm{CL}_{\mathbb{R}}\left(C_{i}\right),
$$

где $f_{i} \in M U^{*}(\mathrm{pt})$. Тогда получим искомьй эпиморфизм.

Предложение доказано.

\section{СПИСОК ЦИТИРОВАННОЙ ЛИТЕРАТУРЫ}

[1] Краснов В. А. Неравенства Гарнака-Тома для отображений вещественных алгебраических многообразий // Изв. АН СССР. Сер. матем. 1983. Т. 47. № 2. С. 268-297.

[2] Краснов В.А. Об эквивариантных когомологиях Гротендика вещественного алгебраического многообразия и их приложениях // Изв. РАН. Сер. матем. 1994. Т. 58. № 3. С. 36-52.

[3] Краснов В. А. Вещественные алгебраические $G M$-многообразия // Изв. РАН. Сер. матем. 1998. Т. 62. №3. С. 39-66.

[4] Краснов В.А. Вещественные алгебраические многообразия и кобордизмы // Изв. РАН. Сер. матем. (в печати).

[5] Стонг Р. Заметки по теории кобордизмов. М.: Мир, 1973.

Ярославский государственный университет

E-mail: krasnov@uniyar.ac.ru 